Article

\title{
Optimization Design of Electromagnetic Actuator Applied as Fast Tool Servo
}

\author{
Yahui Nie ${ }^{1,2, *}$, Yinfei $\mathrm{Du}^{3}$ and Zhuo $\mathrm{Xu}{ }^{1,2}$ \\ 1 School of Mechanical Engineering, Tianjin University of Technology and Education, Tianjin 300222, China; \\ tzsdxuzhuo@foxmail.com \\ 2 Tianjin Key Laboratory of High Speed Cutting \& Precision Machining, \\ Tianjin University of Technology and Education, Tianjin 300222, China \\ 3 Tianjin Institute of Metrological Supervision and Testing, Tianjin 300192, China; duyinfei521@foxmail.com \\ * Correspondence: zynyh@hotmail.com; Tel.: +86-138-2092-7234
}

Received: 21 June 2017; Accepted: 8 August 2017; Published: 18 August 2017

\begin{abstract}
Fast tool servos (FTS) for single point diamond turning are widely employed for machine optical free-form surfaces. A FTS with a large stroke and a high bandwidth can increase the efficiency of a machine and complexity of a work-piece. In this paper, a FTS driven by a Maxwell electromagnetic actuator is developed to obtain a relatively large stroke and high bandwidth. In this study, a multi-objective optimization model is proposed based on the whole system by considering electromagnetic driving principles, kinematic model, and mechanical model. The proposed optimization model can be applied to solve the parameters of electromagnetic actuators with diverse application requirements. A sequential quadratic programming (SQP) algorithm is implemented to solve the problem. The optimization results are verified through both finite element analysis and experiments. The optimized FTS can produce $49.55 \mu \mathrm{m}$ of full stroke with a frequency response of $3.2 \mathrm{kHz}$.
\end{abstract}

Keywords: electromagnetic actuator; fast tool servo; freeform optics; sequential quadratic programming

\section{Introduction}

Optical free-form surfaces are increasingly used in various areas, such as the aerospace, energy, and life science. The single-point diamond turning method based on the fast tool servo (FTS) system is widely adopted due to the advantages of high accuracy and superior process efficiency [1]. A wide variety of FTSs exist which employ different types of actuators [2]. These FTSs provide different strokes and bandwidths. The stroke of a FTS determines the non-rotationally symmetric component of a desired surface, while the bandwidth determines the productivity, surface complexity, and even the materials that can be processed by the machine [3]. A FTS with a larger stroke (several millimeters) and a higher bandwidth (several thousands of hertz) is necessary to process more complex surfaces of higher amplitude with machines. However, in reality, a large stroke and high bandwidth cannot be implemented at the same time. Therefore, different types of FTSs with different strokes and bandwidths were designed.

FTSs driven by piezoelectric actuators (PZT-FTS) [4,5], FTSs driven by Lorentz forces in the voice-coil motor (VCM-FTS) [6,7] and FTSs driven by Maxwell electromagnetic actuators (MEA-FTS) [8,9] have been widely studied by researchers. Based on different driving methods, different types of flexure-based mechanisms—-such as flexure hinges, leaf springs, and levers-were applied to realize FTSs with specific strokes and bandwidths. Many researchers investigated optimization methods to solve the parameters of the flexures in their specialized FTSs [10-14]. 
Zhu et al. developed a two-dimensional FTS, which can obtain better profile accuracy and surface quality due to its novel adaptive tool servo (ATS) diamond turning method [10]. Yang et al. employed a commercialized PZT actuator to create their FTS [11]. A flexure optimization was performed based on the finite analysis model of commercial software ANSYS in their design. The optimized FTS was able to produce a stroke of $24.6 \mu \mathrm{m}$ at a first resonant frequency of $2192 \mathrm{~Hz}$. Zhu et al. fulfilled their requirements for a FTS by optimizing the parameters of a flexure hinge with the multi-objective method based on the differential evolution algorithm [12]. Their FTS produced a stroke of $10.25 \mu \mathrm{m}$ with a first resonant frequency of $2000 \mathrm{~Hz}$. Zhuang et al. proposed a multi-objective topology optimization method for the design of a compliant mechanism, with the proposed design showing better performance by increasing the effective stroke of FTS in addition to improving the stiffness in the vertical (main cutting force) and horizontal (feed force) directions [13]. Rakuff et al. used finite element analysis to optimize the parameters of their specially designed leaf flexure before adjusting the damping ratio of the FTS system with special experiments [14]. Their FTS completed ultra-precision machining successfully due to its characteristics of a $2 \mathrm{~mm}$ stroke and a bandwidth of $140 \mathrm{~Hz}$. Most researchers focused on optimizing the design of flexures rather than the whole FTS system. However, a typical FTS system consists of both flexures and the actuator producing a driving force as well as the transmission unit. The ultimate performance of FTS depends on the whole system. Hence, both the optimization results and the ultimate performance of the optimized FTS are affected.

A survey of the literature shows that the optimizations of FTS structure were focused on flexures, despite flexures being only one part of the FTS system. The actuator, the tool holder, and other parts have not been considered for optimization in these studies, meaning that previous optimization was not based on the whole system. Thus it can be suggested that FTS has not been fully optimized and its best performance has not been reached.

Compared to PZT-FTS with the limitation of small stroke and VCM-FTS with the limitation of low bandwidth, the MEA-FTS can implement FTS at a larger stroke compared to PZT-FTS (at the same bandwidth) and a higher bandwidth compared to VCM-FTS (at the same stroke) $[15,16]$. According to a series of surveys, a fast tool servo driven by the electromagnetic actuator has the ability to obtain a relatively larger stroke and higher bandwidth compared to PZT-FTS and VCM-FTS. Figure 1 shows the characteristics of a series of FTSs. It shows the advantages of MEA-FTS. Similar conclusions have also been reported by $\mathrm{Lu}$ and Xie $[15,16]$. In this paper, MEA-FTS is studied and a multi-objective optimization model is built based on the driving model of electromagnetic actuator, mechanical structure, and kinematic model. The optimal parameters of the electromagnetic model and mechanical structure are solved. A generalized optimization model of MEA-FTS is proposed and the optimization model can be applied to many types of FTS at a variety of strokes and frequencies.

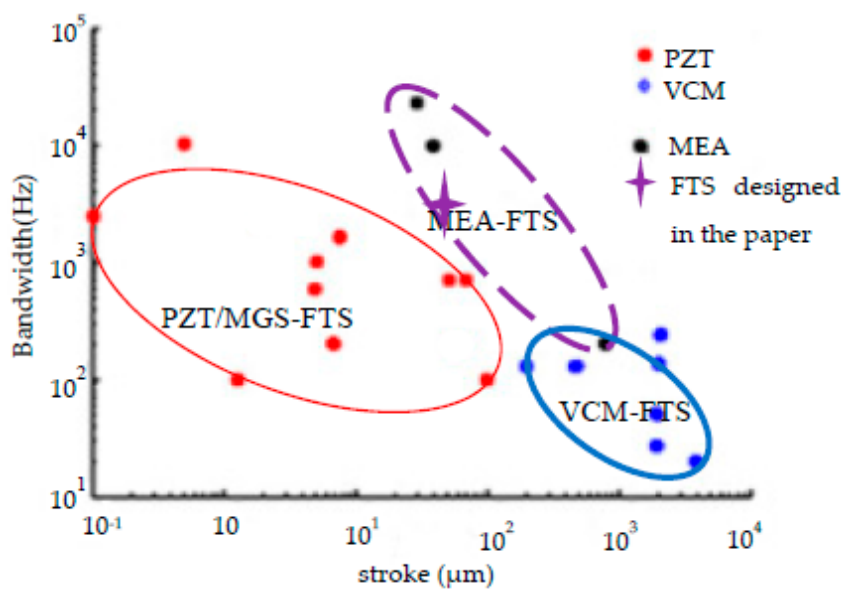

Figure 1. The stroke and bandwidth characteristics of a series of FTS. 


\section{Materials and Methods}

\subsection{Construction of MEA-FTS Model}

\subsubsection{Electromagnetic Driving Model}

A 3D-model of MEA-FTS is shown in Figure 2. MEA-FTS consists of three parts: the frame, electromagnetic part, and moving part. The electromagnetic part produces the actuating force on the armature, while the moving part transforms the actuating force into specific movements.

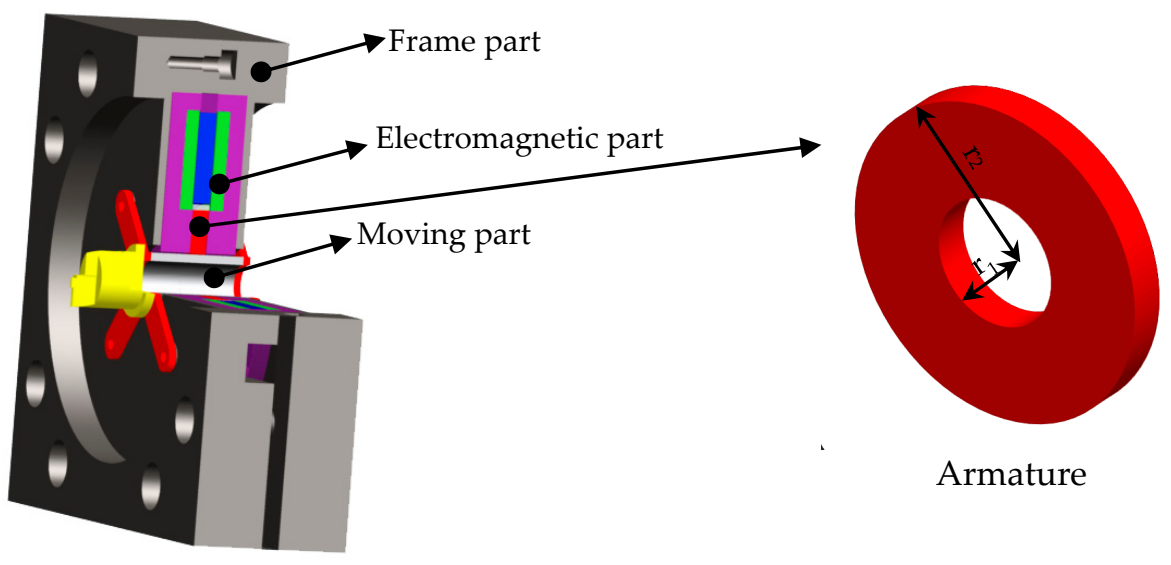

Figure 2. 3D model of MEA-FTS.

The magnetic part consists of yokes, coil windings, a permanent magnet, armature, and air gaps between the yokes and armature. The magnetic part is a rotationally symmetrical 2D-model, which is shown in Figure 3. This magnetic part is used to analyze the electromagnetic principles. The permanent magnet produces a magnetic flux, which is shown in Figure 3 as the biasing flux. The exciting flux, shown in Figure 3, is produced by currents in the coil windings. MEA-FTS is driven by the Maxwell electromagnetic force, which involves the electromagnetic tension in air gaps [13]. The magnetic field in both air gaps consists of a flux density $\left(B_{B}\right)$ excited by the permanent magnet and a flux density $\left(B_{E}\right)$ excited by the currents in coil windings. Assuming that the flux density in yokes is not saturated, we define the Maxwell electromagnetic force acting on the left side of armature as $F_{l}$; the Maxwell electromagnetic force acting on the right side of armature as $F_{r}$; the flux density in the left air gap as $B_{l}$; and the flux density in the right air gap as $B_{r}$. The magnetic fields in both air gaps are consistent with the superposition theorem. Therefore, we can conclude the following: $B_{r}=B_{B r}+B_{E}$ and $B_{l}=B_{B l}-B_{E}$. We define $B_{B r}$ as the flux density excited by the permanent magnet in the right air gap and $B_{B l}$ as the flux density excited by the permanent magnet in the left air gap. When the displacement of the armature is $z$, the actuating Maxwell force can be calculated according to the equation [17]

$$
F=F_{r}-F_{l}=\frac{S}{2 \mu_{0}}\left(B_{r}^{2}-B_{l}^{2}\right)=\frac{S}{2 \mu_{0}}\left(B_{B r}+B_{B l}\right)\left(2 B_{E}+\left(B_{B r}-B_{B l}\right)\right)=\frac{S}{\mu_{0}} B_{B}\left(\frac{\mu_{0} N I}{z_{0}}+\frac{2 z}{z_{0}} B_{B}\right)
$$

where $z_{0}$ is the air gap when the armature is in the middle of the model; $z$ is the displacement of the armature (unit: $\mathrm{m}$ ); $B_{B}$ is the magnetic flux density generated by the permanent magnet (unit: T); $S$ is the yoke pole face area at each working gap (unit: $\left.\mathrm{m}^{2}\right) ; \mu_{0}$ is the permeability of air $\left(\mu_{0}=4 \pi \times 10^{-7} \mathrm{~N} / \mathrm{A}^{2}\right)$; $N$ is the number of turns in the coils; and $I$ is the exciting current (unit: A). 


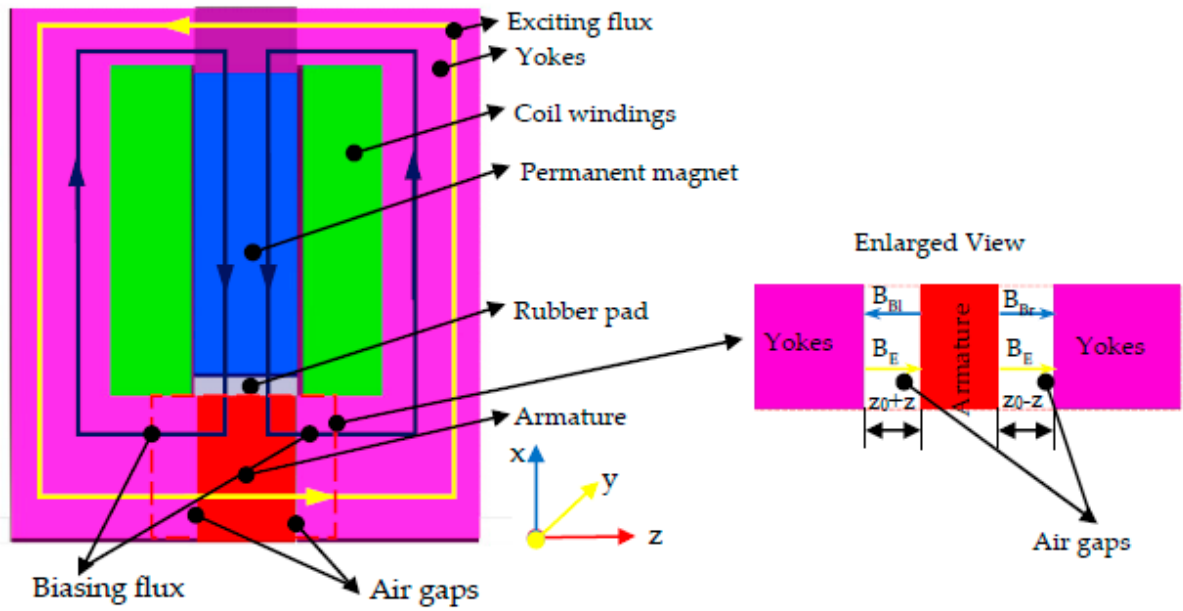

Figure 3. 2D model of the magnetic part.

The formula of the maximum driving force should be derived to estimate the stroke of MEA-FTS. According to Equation (1), the maximum driving force can be derived through Equation (2)

$$
\begin{aligned}
& F_{\max }=\max \left\{\frac{S}{2 \mu_{0}} \cdot\left(B_{B r}+B_{B l}\right) \cdot\left(2 B_{E}+\left(B_{B r}-B_{B l}\right)\right)\right\} \\
& \text { s.t. }\left\{\begin{array}{l}
0 \leq B_{B r}+B_{E} \leq B_{\text {sat }} \\
0 \leq B_{B l}-B_{E} \leq B_{\text {sat }}
\end{array}\right.
\end{aligned}
$$

The actuating force reaches its maximum when the flux density in the yoke becomes saturated ( $B=B_{\text {sat }}$, where $B_{\text {sat }}$ is the saturated flux density) with maximum displacement of the armature $\left(z=z_{0}\right.$, where $\left.B_{B r}=B_{B l}\right)$. We define the outer radius of the armature as $r_{2}$ and the inner radius of armature as $r_{1}$. Therefore, the yoke pole face area at each working gap is $S=\pi\left(r_{2}{ }^{2}-r_{1}{ }^{2}\right)$. The maximum actuating force can be derived as Equation (3)

$$
F_{\max }=\frac{B_{\text {sat }}^{2} \cdot \pi\left(r_{2}^{2}-r_{1}^{2}\right)}{\mu_{0}}
$$

According to Equation (3), the maximum driving force is proportional to the squared value of $B_{\text {sat }}$ squared. Thus, the Kool $\mathrm{M} \mu$ is chosen according to the magnetic material of the yokes and armature. The saturation flux density of Kool $\mathrm{M} \mu$ is $1.4 \mathrm{~T}$, while the relative permeability is 125 .

\subsubsection{Mechanic Model}

FTS systems driven by electromagnetic actuators have the potential to achieve a relatively larger stroke and higher bandwidth, but proper flexure designs play an important role in obtaining better performance. Two symmetric leaf flexures are applied in the mechanical design model in order to compensate thermal variations and mechanical disturbances. The moving part of the MEA-FTS, shown in Figure 4, consists of the armature, two symmetric leaf flexures, a connector, and a specially designed tool holder. The armature driven by the Maxwell electromagnetic force produces displacement, with flexures and the tool holder being connected to armature by the connector. The eight feet of the two flexures are fixed on the frames through bolts. When the armature is driven by the Maxwell electromagnetic force, the force passes to the flexures, resulting in elastic deformation. Following this, the displacement force is passed onto the diamond tool. 


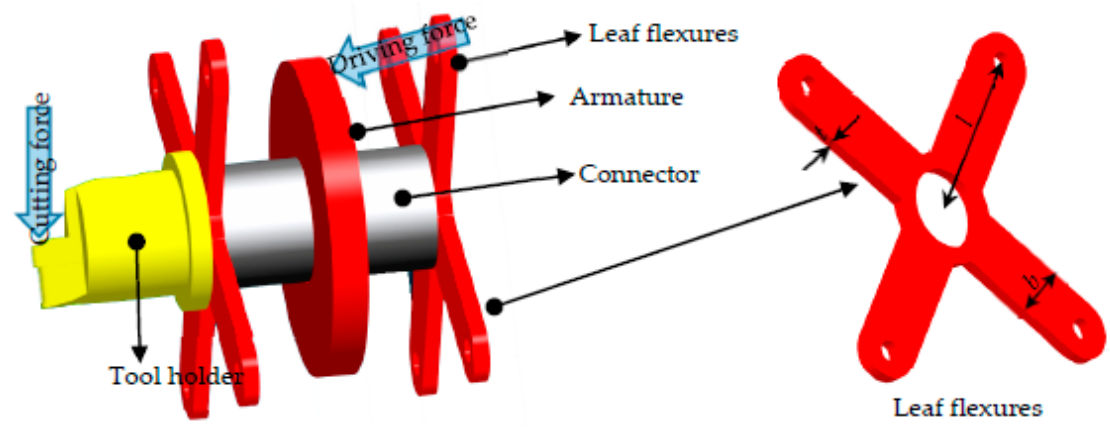

Figure 4. Moving part of MEA-FTS.

When the MEA-FTS is used for machining, the moving part of MEA-FTS will be subjected to two forces: the Maxwell electromagnetic force acting on the armature and the cutting force acting on the diamond tool. The force diagram is shown in Figure 4. In ultra-precision machining, the depth of cut is commonly very shallow (1-3 $\mu \mathrm{m}$ during the finishing cut) and thus, the cutting force (smaller than $10 \mathrm{~N}$ ) can be neglected as being trivial compared to the driving force [18]. Thus, we ignore the effect of the cutting force during optimization of the design of the moving and electromagnetic parts of MEA-FTS in this study.

The effects of stroke, bandwidth, and rising time are determined by the mechanical stiffness. Assuming that the leaf flexures deform within the range of elastic deformation (the assumption is guaranteed through constraints) and according to Hooke's law, the mechanical model of MEA-FTS is a cantilever structure. Therefore, the stiffness of MEA-FTS can be obtained by [19]

$$
k=2 \times 4 \times\left(3 \cdot E \cdot \frac{b \cdot t^{3}}{12 \cdot l^{3}}\right)=\frac{2 \cdot E \cdot b \cdot t^{3}}{l^{3}}
$$

where $E$ is the Young's modulus of leaf flexures; while $l, b$, and $t$ represent the length, width, and thickness of the flexure, respectively.

\subsubsection{Driving Response Model}

Assuming that MEA-FTS is a quadratic damping system, an equivalent kinematic model of MEA-FTS can be obtained, which is shown in Figure 5. Assuming that the damping coefficient is $\mu$, the weight of the movement part in the system is $m$ and according to Newton's law, the dynamic equilibrium equation of the actuator at time $t$ can be obtained using the equation

$$
m \cdot z(t)^{\prime \prime}+\mu \cdot z(t)^{\prime}+k \cdot z(t)=F(t)
$$

where $z(t)$ is the displacement of armature at time $t$. Assuming that the deformation of connectors is zero, the displacement of the tool is $z(t) ; z^{\prime \prime}=\mathrm{d}^{2} z(t) / \mathrm{d} t^{2}$ is the acceleration of the moving part; and $z^{\prime}=\mathrm{d} z(t) / \mathrm{d} t$ is the velocity of the moving part.

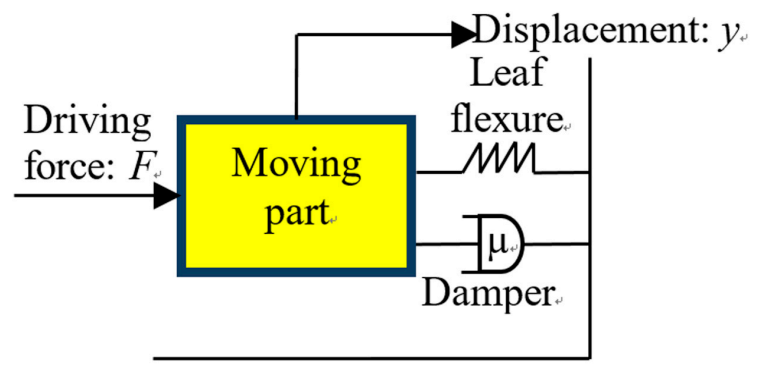

Figure 5. Equivalent kinematic model of MEA-FTS. 
Following this, the transient response of the MEA-FTS can be deduced as [20]

$$
z(t)=\frac{\frac{A}{\mu_{0}} B_{B} \frac{\mu_{0} N I}{z_{0}}}{k_{z}}\left[1-\frac{e^{-\xi \omega_{n} t}}{\sqrt{1-\xi^{2}}} \sin \left(\omega_{n} \sqrt{1-\xi^{2}} t+\arctan \frac{\sqrt{1-\xi^{2}}}{\xi}\right)\right]
$$

where, $k_{z}=k-\frac{B_{B}}{y_{0}} ; \omega_{n}=\sqrt{\frac{k_{y}}{m}}$; and $\xi=\frac{\mu}{2 m \omega_{n}}$.

The first-order resonant frequency of the system can be expressed as [21]

$$
f=\frac{\omega_{n} \sqrt{1-\xi^{2}}}{2 \pi}=\frac{\sqrt{4 m k_{z}-\mu^{2}}}{4 \pi m}
$$

The response time of the system can be expressed as

$$
t_{d}=\frac{2 m+0.7 \frac{\mu}{\omega_{n}}}{2 m \omega_{n}}=\frac{2 m \omega_{n}+0.7 \mu}{2 m \omega_{n}^{2}}
$$

The response time reflects the response speed of MEA-FTS. The response time determines the controlling bandwidth if the system has a closed-loop control. The bandwidth is inversely related to the response time. Based on Equations (7) and (8), the response time $t_{d}$ is linearly related to the reciprocal of the first-order resonant frequency $f$. Optimization of either will obtain the optimized bandwidth of MEA-FTS. Moreover, the first-resonant frequency should be the priority, because severe damage will occur at the first-resonant frequency. Thus, the bandwidth of the system will be measured as the first-order resonant frequency in this paper.

\subsubsection{Construction and Implementation of Optimization Model}

Objective Functions of the Problem

The machining characteristic of a FTS depends on its stroke and bandwidth. The stroke of a FTS determines the sagittal height of a free-form surface, while the bandwidth determines the machining speed and surface complexity. Therefore, in the optimization design, a MEA-FTS with a larger stroke and higher bandwidth would be ideal to obtain.

Substituting Equations (1) and (4) into the equations of Hooke's law, the stroke of the MEA-FTS can be obtained by

$$
\delta=2 \cdot \frac{F_{\max }}{k}=\left(\frac{B_{s a t}^{2} \cdot \pi\left(r_{2}^{2}-r_{1}^{2}\right) \cdot l^{3}}{4 \mu_{0} E b t^{3}}\right)
$$

where, $r_{2}$ is the outer diameter of the armature; and $r_{1}$ is the inner diameter of the armature.

According to the analysis of Section 2.1.3 and Equation (7), the first-resonant frequency of MEA-FTS can be expressed as

$$
f=\frac{\sqrt{4 m k_{z}-\mu^{2}}}{4 \pi m}=\frac{\sqrt{4\left(\pi \rho \cdot t_{a}\left(r_{2}^{2}-r_{1}^{2}\right)+m_{0}\right) \frac{E \cdot b \cdot t^{3}}{l^{3}}-\mu^{2}}}{4 \pi \cdot\left(\pi \rho \cdot t_{a}\left(r_{2}^{2}-r_{1}^{2}\right)+m_{0}\right)}
$$

where, $t_{a}$ is the thickness of armature, $\rho$ is the density of magnetic material.

According to Equations (9) and (10), it is concluded that stroke and bandwidth are interrelated. Furthermore, both of them are affected by dimensions of the armature and the dimensions of the designed flexure. Most of the parameters (e.g., $r_{2}, r_{1}, t, b, l$ ) have counter effects on the stroke and bandwidth. For example, the parameters of $r_{2}$ and $l$ should be as large as possible to obtain a larger stroke, however larger parameters of $r_{2}$ and $l$ will lead to smaller bandwidth. Thus it is impossible to obtain an FTS with large stroke and high bandwidth. There must be a trade-off between the stroke and bandwidth. 
Therefore, the maximum stroke and maximum bandwidth are set as goals. In order to solve the problem easily, the objective functions are written as

$$
\begin{gathered}
(M O P) \quad \min \left\{F(x): x \in R^{6}, F(x)=\left(F_{1}(x), F_{2}(x)\right)^{T}\right\} \\
\text { s.t. } \quad\left\{\begin{array}{c}
F_{1}(x)=\frac{1}{\delta}=\left(\frac{4 \mu_{0} E \cdot x_{3} \cdot x_{2}^{3}}{B_{\text {sat }}^{2} \cdot \pi\left(x_{6}^{2}-x_{5}^{2}\right) \cdot x_{4}^{3}}\right) \\
F_{2}(x)=\frac{1}{f}=\frac{4 \pi \cdot\left(\pi \rho \cdot t_{a}\left(r_{2}^{2}-r_{1}^{2}\right)+m_{0}\right)}{\sqrt{4\left(\pi \rho \cdot t_{a}\left(r_{2}^{2}-r_{1}^{2}\right)+m_{0}\right) \frac{E \cdot b^{3} t^{3}}{l^{3}}-\mu^{2}}}
\end{array}\right.
\end{gathered}
$$

where $x=\left(x_{6}, x_{5}, x_{4}, x_{3}, x_{2}, x_{1}\right)^{\mathrm{T}}=\left(r_{2}, r_{1}, l, b, t, t_{\mathrm{a}}\right)$ are the decision variables; while $F_{1}(x)$ and $F_{2}(x)$ are continuously differentiable (highly non-linear) functions in their defined domain. All the decision variables are annotated in Figure 6.

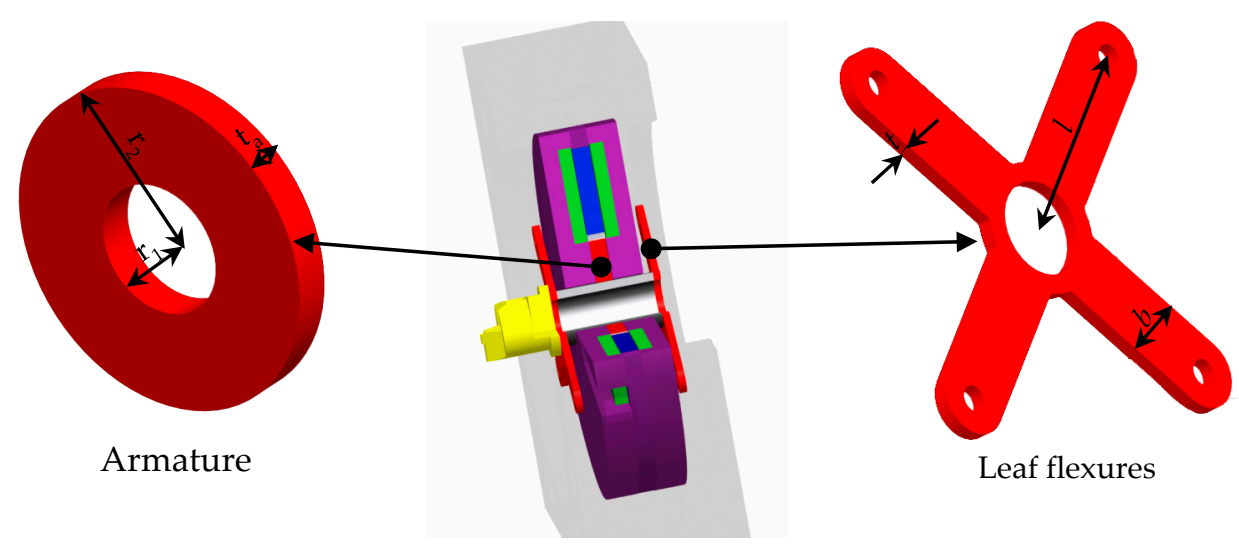

Figure 6. Annotation of decision variables.

Constraints of the Problem

In the optimization model, the variables should be solved under several constraints. These constraints are considered from several aspects as follows:

(1) Allowable stress of flexure

The kinematic model is constructed based on the elastic deformation assumption. Therefore, an inelastic deformation should be avoided. The stress constraint inequality can be expressed as

$$
\sigma_{\max }=\frac{3 \cdot F_{\max } \cdot l^{2}}{b \cdot t^{3}}=\frac{3 \pi \cdot B_{s a t}^{2} \cdot\left(r_{2}^{2}-r_{1}^{2}\right) \cdot l^{2}}{\mu_{0} \cdot b \cdot t^{3}} \leq \frac{\sigma_{\lim }}{n_{s 1}}
$$

where $\sigma_{\max }$ is max stress in the flexure, $\sigma_{\text {lim }}$ is the allowable stress of material, and $n_{s 1}$ is a safety factor.

(2) Magnetic dimensional constraints

In this design, the Maxwell electromagnetic force is established under the conditions that the magnetic field in the working gaps are distributed uniformly, and the flux lines are in accord with the magnetic circuits. Assuming that the thickness of armature is $t_{a}$, the constraint inequalities are

$$
\left\{\begin{array}{l}
t_{a}=n_{s 2} \cdot 10 \cdot \delta ; \\
r_{2}-r_{1} \geq n_{s 3} \cdot 10 \cdot l_{f r}
\end{array}\right.
$$

where $n_{s 2}, n_{s 3}$ are the safety factors; while $l_{f r}$ is the length of gap between the armature and permanent magnet. 
(3) Dimension and processing constraints

In the mechanical design, the dimension and processing constraints should also be considered. Due to the dimensions of our sensor used for measuring the displacement of the tool holder, the inner radius of the armature should be larger than $5 \mathrm{~mm}$. Thus, the constraint inequality should be $\left(5-r_{2}\right) \leq 0$. Obviously, the outer radius of the armature should be larger than its inner radius. Thus, the constraint inequality should be $\left(r_{2}-r_{1}\right) \leq 0$. Due to the processing technology of the magnetic material, the outer radius of the armature should be smaller than $30 \mathrm{~mm}$. Thus, the constraint inequality should be $\left(r_{1}-30\right) \leq 0$. Considering the processing technology and difficulty of fabrication, the thickness of flexure is restricted to being larger than $0.5 \mathrm{~mm}$. Thus, the constraint inequality should be $t \geq 0.5$. With the limitation for the dimensions of both the sensor and diamond turning machine, the length of flexure is restricted to be within the range of $10 \mathrm{~mm}$ to $50 \mathrm{~mm}$. Thus, the constraint inequality should be $10 \leq l \leq 50$.

In general, the constraint inequalities of the problem are

$$
G(x)=\left\{G_{9}(x), G_{8}(x), G_{7}(x), G_{6}(x), G_{5}(x), G_{4}(x), G_{3}(x), G_{2}(x), G_{1}(x)\right\}^{T}
$$

where $G_{9}(x)=\frac{3 \pi \cdot B_{s a t}^{2} \cdot\left(x_{6}^{2}-x_{5}^{2}\right) \cdot x_{4}^{2}}{\mu_{0} \cdot x_{3} \cdot x_{2}^{3}} \leq \frac{\sigma_{\lim }}{n_{s 1}} ; G_{8}(x)=x_{6}-x_{5}-n_{s 3} \times 10 \times 1 \mathrm{fr} \leq 0 ; G_{7}(x)=5-x_{5} \leq 0$; $G_{6}(x)=x_{6}-x_{5} \leq 0 ; G_{5}(x)=x_{6}-30 \leq 0 ; G_{4}(x)=0.5-x_{3} \leq 0 ; G_{3}(x)=x_{2}-5 \leq 0 ; G_{2}(x)=10-x_{4} \leq 0$; and $G_{1}(x)=x_{4}-50 \leq 0$.

\section{Construction of Optimization Model}

In the optimization model for MEA-FTS, the stroke and bandwidth are chosen as the optimization objectives. Both the stroke and the first-resonant frequency depend on the parameters of the magnetic circuit structure, flexure structure, and dimensions of the armature [22]. The flexure dimensions $(b, l$, and $t)$, the armature dimensions $\left(r_{1}\right.$ and $\left.r_{2}\right)$ and the magnetic circuit dimensions $\left(t_{a}\right)$ are the decision variables. The optimization problem (11) can be described as

$$
\begin{array}{ll}
\min & F(x) \\
\text { s.t. } & G(x) \leq 0
\end{array}
$$

where $F(x)$ is a continuous function mapping from 6D real space to a 2D real space; and $G(x)$ is a continuous function mapping from a $6 \mathrm{D}$ real space to a 9D real space. The definition domain of the problem should be $\left\{x: \mid x_{6} \neq x_{5}, x_{4} \neq 0,2 \cdot E \cdot x_{3} \cdot x_{2}{ }^{3} \cdot y_{0} \neq B_{B} \cdot x_{4}{ }^{3}\right\}$. Thus, $F(x)$ is a non-linear continuous function, while $G(x)$ is also a non-linear continuous function. We define $f^{*}$ and $\delta^{*}$ as the expected bandwidth and stroke, respectively.

The MEA-FTS optimization problem concerns the minimization of a set of objectives while simultaneously satisfying all types of constraints. The objective function is consisted of two non-linear functions. Both are the multi-variable functions of the same parameters for MEA-FTS. The MEA-FTS optimization problem addresses the problem of reducing two non-linear functions below two goals. The design parameters of the magnetic structure and mechanical structure can be obtained. The optimization problem of MEA-FTS is a non-linear multi-objective optimization problem with several constraints. In this paper, we propose that the original problem can be solved through the SQP (sequential quadratic programming) algorithm after reformulation of the problem.

\subsection{Implementation of the Problem}

\subsubsection{Reformulation of the Problem}

In this paper, the SQP algorithm is adopted to solve the problem. The goal attainment problem formulation allows the objectives to be overachieved. Therefore, the mechanical dimensions can be relatively imprecise when related to the calculated solutions. This advantage allows for the design of 
mechanical structures with the consideration of limitation of equipment and processing technique [23]. Therefore, the problem is reformulated as a goal attainment problem. We define weighting ratios as $W=\left[W_{1}, W_{2}\right]$, with the problem then being reformulated as

$$
\begin{array}{ll}
\min & \psi(x)=\frac{F_{1}(x)-\frac{1}{\delta^{*}}}{w_{1}}+\frac{F_{2}(x)-\frac{1}{f^{*}}}{w_{2}} \\
\text { s.t. } & G(x) \leq 0
\end{array}
$$

Following this, the problem can be solved using the SQP algorithm. The SQP algorithms represent in the state-of-the-art thinking in non-linear programming algorithms. This type of algorithm has superior performance in terms of efficiency, accuracy, and success [24,25]. Equation (16) concerns the minimization of a set of objectives while simultaneously satisfying all types of constraints. The objective function consists of two non-linear functions. Both are the multi-variable functions of the same parameters for MEA-FTS. The inequalities of constraints are non-linear. SQP algorithm has advantages to solve nonlinear constrained optimization problems due to the super-linear and quadratic convergence rate of sequential quadratic programming algorithm [26]. The MEA-FTS optimization problem addresses the problem of reducing two non-linear functions to stay below two goals. The design parameters of the magnetic structure and mechanical structure can be obtained. The optimization problem of MEF-FTS is a non-linear multi-objective optimization problem with several constraints. In this paper, we solve our problem using the SQP method combined with the active set technology [27].

\subsubsection{Solution of the Problem}

In this paper, we solve our problem using SQP method. The main idea of using SQP algorithm to solve a non-linear optimization problem is that, at each major iteration, an approximation solution is used to simplify the original non-linear problem. Following this, a new linear quadratic problem is obtained. If the optimal solution can be obtained after this, the solution is the optimal solution of the original problem. Otherwise, the new solution is used as a new approximation solution and the iteration continues until the solution of the problem is obtained [22]. Therefore, when Equation (16) is solved, a positive definite approximation of the Hessian matrix of Lagrangian should be calculated by any quasi-Newton methods such as the BFGS (Broyden, Fletcher, Goldfarb and Shanno's Quasi-Newton) method. Then the quadratic sub-problem can be formulated as

$$
\begin{aligned}
& \min \frac{1}{2} d^{T} H_{k} d+\forall f\left(x_{k}\right)^{T} d, \\
& \forall g_{i} f\left(x_{k}\right)^{T} d+g_{i}\left(x_{k}\right) \leq 0, i=1,2
\end{aligned}
$$

This subproblem can be solved using the Wilson-Han-Powell (WHP) algorithm. The solution is used to form a new iteration: $\left(X_{k+1}=X_{k}+\alpha_{k} d_{k}\right)$, where the step length $\alpha_{\mathrm{k}}$ should be calculated by an appropriate line search procedure so that a sufficient decrease can be obtained [25]. Following this, the solution of Equation (16) can be obtained after several iterations.

In order to solve problem (16), proper goals should be set. The stroke and bandwidth of MEA-FTS are in conflict with each other. Both of them are constrained by the power of MEA-FTS as follows

$$
P=V \cdot I=N I \cdot\left(\frac{V}{N}\right)=\frac{B_{s a t} \cdot \delta}{\mu_{0}} \cdot\left(2 \pi f \cdot S \cdot B_{\text {sat }}\right)
$$

The power of MEA-FTSs are designed to be $3 \mathrm{~kW}$. Based on typical applications, various goals for bandwidths and strokes are set. The solutions for each set of goals can be obtained using the SQP methods. Table 1 shows several solutions with various sets of typical goals, where $f^{*}$ represents the desired bandwidth; $\delta^{*}$ represents the desired stroke; $f$ represents the calculated value of bandwidth; and $\delta$ is the calculated value of stroke. Based on those results. 
Table 1. Solutions with various sets of typical goals.

\begin{tabular}{ccccccccc}
\hline$f^{*}(\mathbf{H z})$ & $\delta^{*}(\mu \mathrm{m})$ & $f \mathbf{H z})$ & $\delta(\mu \mathbf{m})$ & $t(\mathbf{m m})$ & $\boldsymbol{b}(\mathbf{m m})$ & $\boldsymbol{l}(\mathbf{m m})$ & $\boldsymbol{r}_{\mathbf{1}}(\mathbf{m m})$ & $\boldsymbol{r}_{\mathbf{2}}(\mathbf{m m})$ \\
\hline $\begin{array}{c}\text { target } \\
\text { bandwidth }\end{array}$ & $\begin{array}{c}\text { target } \\
\text { stroke }\end{array}$ & $\begin{array}{c}\text { optimized } \\
\text { bandwidth }\end{array}$ & $\begin{array}{l}\text { optimized } \\
\text { stroke }\end{array}$ & $\begin{array}{l}\text { optimized } \\
\text { thickness } \\
\text { of flexure }\end{array}$ & $\begin{array}{c}\text { optimized } \\
\text { width of } \\
\text { flexure }\end{array}$ & $\begin{array}{c}\text { optimized } \\
\text { length of } \\
\text { flexure }\end{array}$ & $\begin{array}{c}\text { optimized } \\
\text { outer radius }\end{array}$ & $\begin{array}{c}\text { optimized } \\
\text { inner radius } \\
\text { of armature }\end{array}$ \\
500 & 250 & 786.9 & 127.6 & 1.4 & 9.8 & 17.8 & 3.5 & 14.9 \\
1000 & 100 & 999.4 & 99.4 & 1.1 & 4.5 & 10 & 3.8 & 15 \\
2000 & 50 & $1.969 \mathrm{k}$ & 49.2 & 1.4 & 5.4 & 10 & 5 & 15 \\
3000 & 30 & $3.057 \mathrm{k}$ & 30.57 & 1.5 & 6.1 & 10 & 5 & 15 \\
3500 & 25 & $3.584 \mathrm{k}$ & 25.6 & 1.6 & 6.4 & 10 & 5 & 15 \\
5000 & 18 & $4.925 \mathrm{k}$ & 17.73 & 1.7 & 7 & 10 & 5 & 15 \\
\hline
\end{tabular}

According to the solutions, it can be concluded that MEA-FTS can be applied to realize FTS with different bandwidths and strokes to complete different types of workpieces. In this paper, MEA-FTS is designed to manufacture optical free-form surfaces with brittle materials. Based on our formal processing tasks and surveys, the optimization goals with a stroke of $50 \mu \mathrm{m}$ and a first-resonant frequency of $3.5 \mathrm{kHz}$ are chosen for building our MEA-FTS. The optimized MEA-FTS can process lens-arrays on brittle materials. For example, it can process the $50 \times 50$ lens-arrays with an aperture of $100 \mathrm{~mm}$ and sagittal height of $50 \mu \mathrm{m}$. According to the calculation of the SQP algorithm, the MEA-FTS should have a stroke of $51.2 \mu \mathrm{m}$ and bandwidth of $3.584 \mathrm{kHz}$. The model of the MEA-FTS with optimized parameters then can subsequently be constructed. The goal attainment problem formulation allows the objectives to be overachieved. Therefore, the mechanical dimensions can be relatively imprecise with regard to the calculated solutions. This advantage allows for designing of the mechanical structures with the consideration of limitation of equipment and processing technique.

\section{Results and Discussion}

\subsection{Finite Element Analysis Validation}

\subsubsection{Finite Element Analysis for Electromagnetic Field}

In order to verify the optimized results, electromagnetic finite element analysis (FEA) was employed. The electromagnetic 3-D model with optimized parameters was built. The 3-D model is rotationally symmetrical and thus, a 2-D simulation model is used in the finite element analysis, which is shown in Figure 3. Following this, the model was meshed with finite elements. NdFeB was loaded as the permanent magnet, which has an intrinsic coercivity of $1110 \mathrm{kA} / \mathrm{m}$ and a remanence flux density of $1.22 \mathrm{~T}$. The exciting current was a sine wave with a frequency of $3.5 \mathrm{kHz}$ and an amplitude of $160 \mathrm{~A}$. Kool $\mathrm{M \mu}$ is chosen as the magnetic material of yokes and armature. The saturation flux density of Kool $\mathrm{M \mu}$ is $1.4 \mathrm{~T}$, the relative permeability is 125 . The boundary of this analysis is the natural boundary. The commercial software Maxwell is employed. The results can be solved.

Following this, the FEA results shown in Figure 7 can be obtained. The FEA can provide designers with the distributions of magnetic flux density and driving force. The distribution results indicated that the flux lines are consistent with the magnetic circuit designs and parameters. According to the FE analysis, the maximum driving force can be calculated as: $F_{\max }=2 \pi \times 35.592=223.63 \mathrm{~N}$. The weight of the moving part is measured to be $30.71 \mathrm{~g}$. This driving force can provide the FTS armature with a maximum driving acceleration of $\mathrm{a}=F_{\max } / \mathrm{m}=223.63 \mathrm{~N} / 30.71 \mathrm{~g}=305 \mathrm{G}\left(\mathrm{G}=9.8 \mathrm{~m} / \mathrm{s}^{2}\right)$. 


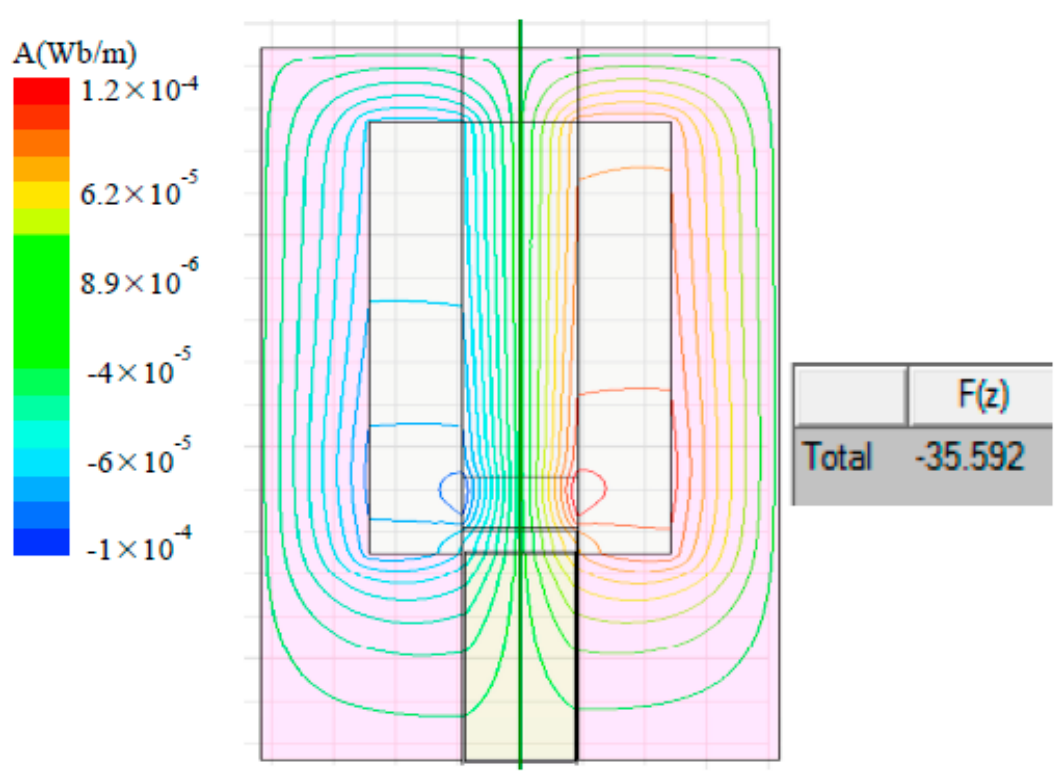

Figure 7. Magnetic flux lines in the MEA-FTS model.

\subsubsection{Finite Element Analysis for Mechanical Structure}

Based on the optimization results, a 3-D mechanical model was built to evaluate the stroke, stress distribution, and first-resonant frequency of the optimized MEA-FTS. The driving force which was obtained in the finite element analysis for electromagnetic field was loaded. According to the FEA results of the mechanical structure shown in Figure 8, it is concluded that: (1) the stroke of MEA-FTS is $53.4 \mu \mathrm{m}$ as shown in Figure 8a, while the first-resonant frequency is $3.857 \mathrm{kHz}$ as shown in Figure 8c. Although the value of the FEA result is slightly lower than that of the optimized result, the design objectives of the optimization can still be satisfied. It can be speculated that firstly, the compromise with processing limits on dimensions leads to a difference between the optimization results and FEA results in the designs of mechanical structures. Secondly, the parts are considered as ideal rigid points in the optimization model, while the parts are considered as thousands of finite elements in the FEA model. (2) The distribution of equivalent stress is shown in Figure $8 \mathrm{~b}$. Therefore, it can be concluded that the stress mainly distributes in two flexures. Furthermore, there would be a stress concentration problem in the contact region between the flexure and the fixing bolt. Considering that the maximum stress in the MEA-FTS model is $277.42 \mathrm{MPa}$, a Mn65 spring steel is chosen as the flexure material.

According to the mechanical FEA results, the optimization model is able to achieve the design goals. The MEA-FTS can achieve a stroke of up to $53.4 \mu \mathrm{m}$ (shown in Figure 8a) and retains a bandwidth of $3.8 \mathrm{kHz}$ (shown in Figure 8c) at the same time. It cannot be ignored that the second resonant frequency is $25 \mathrm{kHz}$, which is shown in Figure 8c. Thus, if a proper control method is designed, the bandwidth of MEA-FTS can be extended to about $25 \mathrm{kHz}$. 


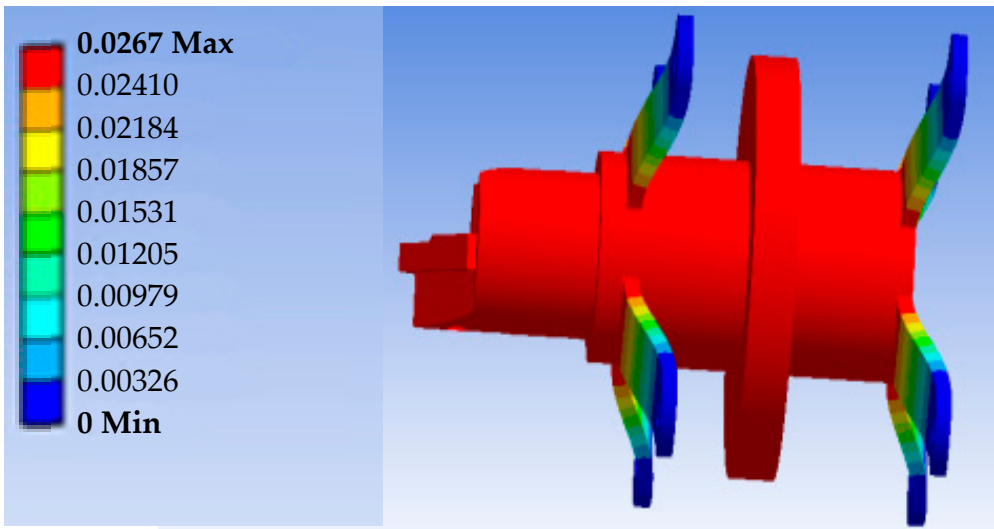

(a) Maximum displacement of one side

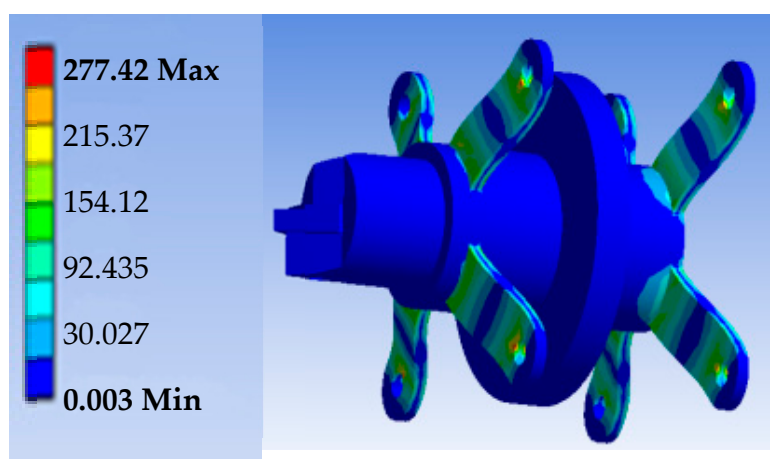

(b) Equivalent stress distribution

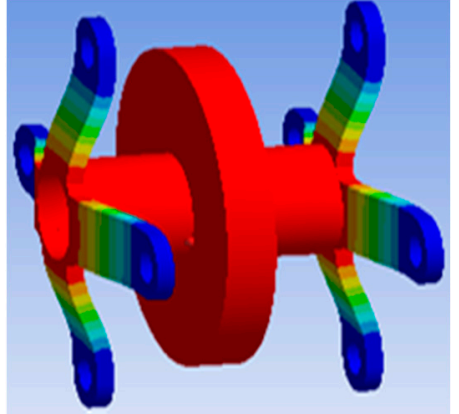

Fisrt mode $3857.7 \mathrm{~Hz}$

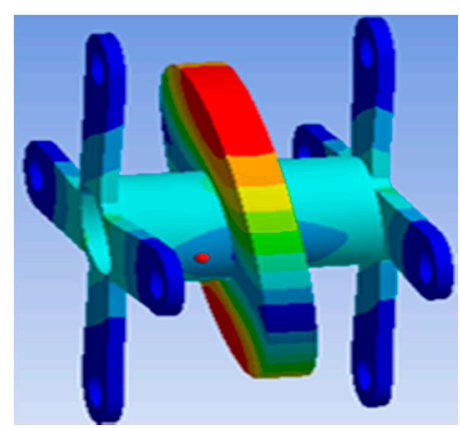

Second mode $25,095 \mathrm{~Hz}$

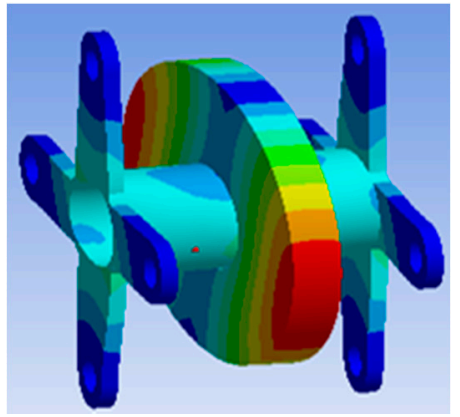

Third mode $25,100 \mathrm{~Hz}$

(c) Modal analysis results of MEA-FTS

Figure 8. Mechanical FEA results.

\subsection{Experiment Tests}

Verification experiments were carried out to validate the optimization results of MEA-FTS. The experiment was performed on a self-designed platform. The platform used for the MEA-FTS experiments is shown in Figure 9. Operational power amplifiers were used to amplify the driving current to reach a current of 160 A. The current was applied to MEA-FTS to generate displacement. The displacements were measured by a laser sensor, which has a resolution of $10 \mathrm{~nm}$, measurement range of $100 \mu \mathrm{m}$, and a bandwidth of $100 \mathrm{kHz}$. 


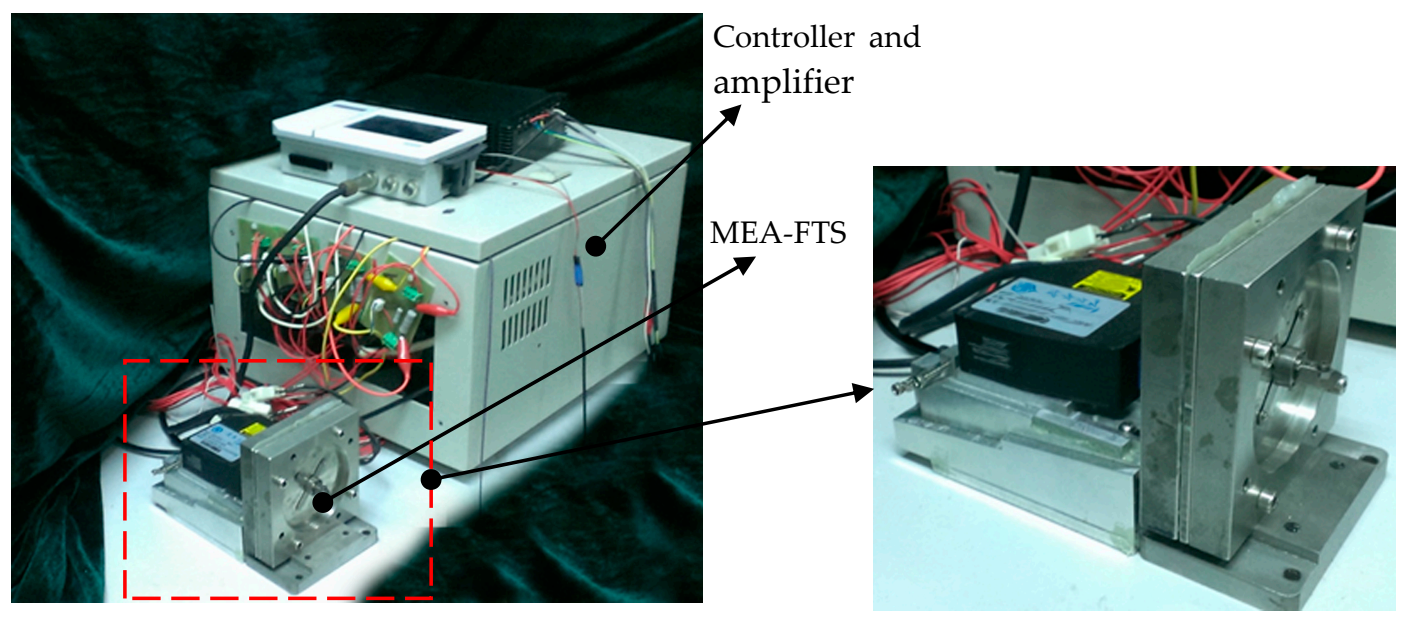

Figure 9. The experiment platform of MEA-FTS.

\subsubsection{Experiment of Displacement}

Experiments were carried out to measure the displacement of MEA-FTS under different driving currents. Based on the results, we can conclude that the displacement of MEA-FTS is linearly related to the driving current, as shown in Figure 10. The maximum displacement in the positive direction is $25.42 \mu \mathrm{m}$ when the maximum positive driving current is loaded. The maximum displacement in the negative direction is $24.13 \mu \mathrm{m}$ when the maximum negative driving current is loaded. The maximum driving current in the exciting coils is $160 \mathrm{~A}$. The stroke of MEA-FTS is $49.55 \mu \mathrm{m}$, which is slightly smaller than the optimization results. This is because we used a rubber pad to adjust the damping ration $\mu$, in this experiment, which was larger than expected.

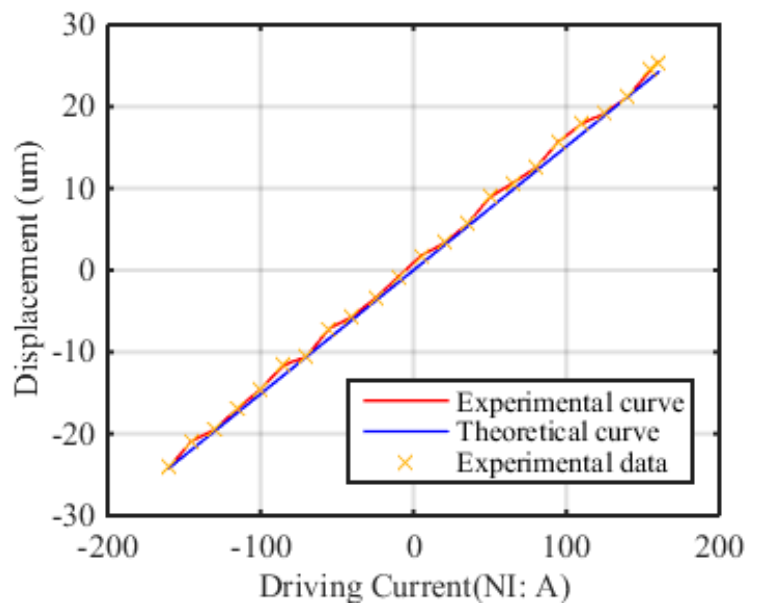

Figure 10. The displacement of MEA-FTS under different driving currents.

\subsubsection{Experiment of Frequency Response}

A frequency experiment was carried out. Sine wave signals with different frequencies ranging from $0.2 \mathrm{~Hz}$ to $100 \mathrm{kHz}$ were amplified to drive the actuator. Following this, the displacement of MEA-FTS was measured by the sensor. The frequency response experimental results are shown in Figure 11. In the results, we found that the stroke became larger after the frequency of $3.2 \mathrm{kHz}$. Therefore, the first resonant frequency (bandwidth) is $3.2 \mathrm{kHz}$. The dropping of the curve from $22 \mathrm{kHz}$ is due to the bandwidth of the power amplifier. 


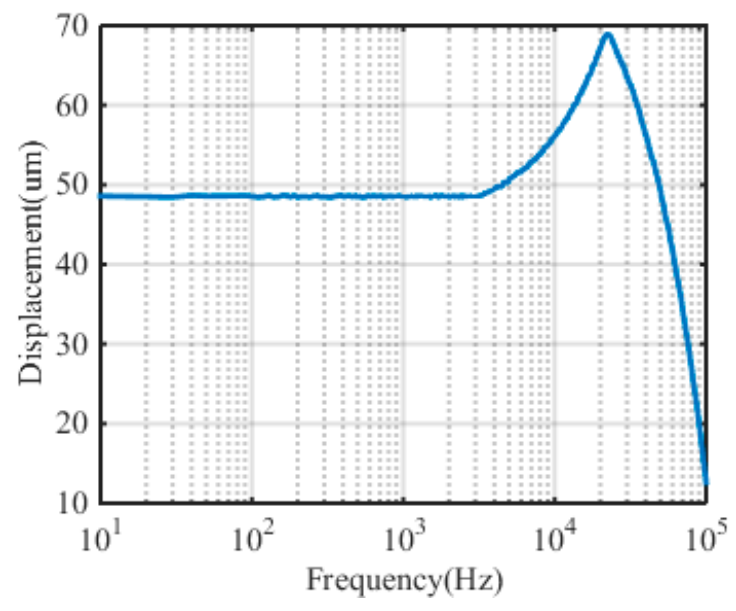

Figure 11. Frequency response curve of MEA-FTS.

\subsubsection{Experiment of Dynamic Response}

A step response test was carried out to measure dynamic characteristics of MEA-FTS. The square wave was used as the exciting signal. The experiment results are shown in Figure 12. Based on experiment results, we can conclude that: (1) MEA-FTS can be accounted as a second order elastic damping system; (2) the response time was $80.48 \mu \mathrm{s}$, so MEA-FTS can be driven to a maximum of $3.11 \mathrm{kHz}$ with closed-loop control; and (3) MEA-FTS had no overshoot in its step response curve. Therefore, when it is applied in free-form machining applications, no over-cut problem will exist.

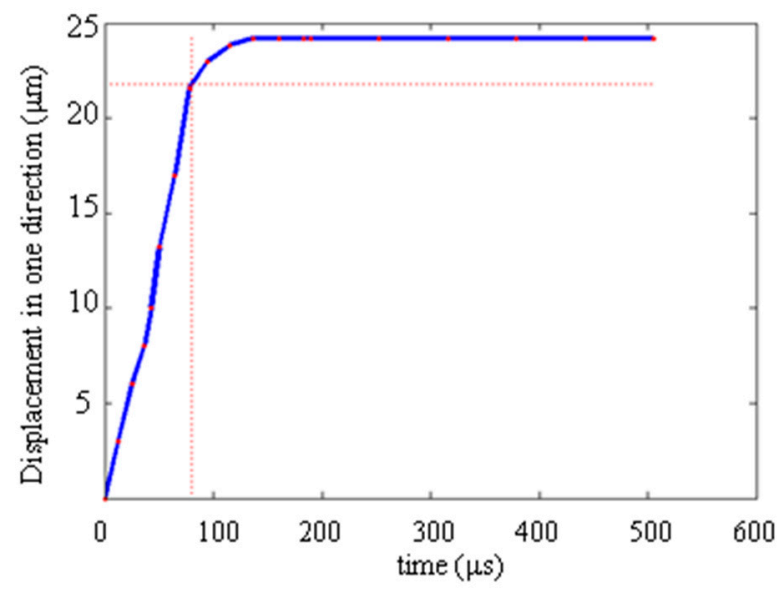

Figure 12. The step response curve of MEA-FTS.

The optimization goals are satisfied based on the experimental results. The optimization model proposed in this paper can be used to obtain design parameters of other FTS with a selected variety of strokes and bandwidths. MEA-FTS can be applied to fabricate free-form optics.

\section{Conclusions}

An optimized MEA-FTS is designed in this paper. The main characteristic parameters of MEA-FTS are chosen as the optimal goals of the model. The optimization problem is built on the electromagnetic model, mechanical model, and kinematic model of the MEA-FTS system under consideration of various constraints. The original problem is reformulated as a goal attainment problem. Following this, the SQP method is introduced to solve this multi-objective non-linear optimization problem. 
Several sets of goals with diverse applications were solved and the design parameters of MEA-FTSs were obtained.

In order to fabricate free-form optics with brittle material, a MEA-FTS was built. Based on the optimization results, the optimized stroke and first resonant natural frequency were $51.2 \mu \mathrm{m}$ and $3.584 \mathrm{kHz}$, respectively. Finite element analysis and experiments were conducted to verify the optimization results. The final bandwidth is $3.2 \mathrm{kHz}$ and the stroke is $49.55 \mu \mathrm{m}$. It can be concluded that the optimization model and SQP method can be used to solve the design parameters for a MEA-FTS.

Acknowledgments: This work was supported by the Tianjin Higher Education Science and Technology Development Fund Project (No. JWK1709, JWK1716) and Tianjin Market and Quality Supervision and Management Commission of Science and Technology Project (No. 2016-W03).

Author Contributions: Yahui Nie performed the optimal design and simulations, the experiments, analyzed the results, and wrote the paper; Yinfei Du contributed with the experiments and analysis; Zhuo Xu created the MEA-FTS.

Conflicts of Interest: The authors declare no conflicts of interest. The founding sponsors had no role in the design of the study; in the collection, analyses, or interpretation of data; in the writing of the manuscript, or in the decision to publish the results.

\section{References}

1. Chapman, G. Ultra-Precision Machining Systems; an Enabling Technology for Perfect Surfaces; Moore Nanotechnology Systems: Keene, NH, USA, 2004; Volume 1, pp. 1-9.

2. Drive Technique Comparisons 2005. Available online: http:/ /www.pecncsu.edu (accessed on 8 October 2011).

3. Kirk, R.; Roblee, J. Freeform Machining with Precitech Servo Tool Options; Precitech Ultra Precision Technology: Berwyn, PA, USA, 2005; Volume 25, pp. 1-10.

4. Physik Instrumente. Fast Tool Servo Precision Machining Piezo Actuator. Available online: http://www.piusa.us (accessed on 8 October 2013).

5. Ma, H.Q.; Tian, J.; Hu, D.J. Development of a fast tool servo in noncircular turning and its control. Mech. Syst. Signal Process. 2013, 41, 705-713. [CrossRef]

6. Kang, D.; Kim, K.; Kim, D.; Shin, J.; Gweon, D.-G.; Jeong, J. Optimal design of high precision XY-scanner with nanometer-level resolution and millimeter-level working range. Mechatronics 2009, 19, 562-570. [CrossRef]

7. Zhou, X.; Hu, L. An improved adaptive feedforward cancellation for tool trajectory tracking in diamond turning of freeform optics. In Proceedings of the 2010 International Conference on Mechanic Automation and Control Engineering, Wuhan, China, 26-28 June 2010; pp. 3590-3593.

8. Gutierrez, H.M.; Ro, P.I. Magnetic servo levitation by sliding-mode control of nonaffine systems with algebraic input invertibility. IEEE Trans. Ind. Electron. 2005, 52, 1449-1455. [CrossRef]

9. Lu, X.D.; Trumper, D.L. Ultrafast tool servos for diamond turning. CIRP Ann. Manuf. Technol. 2005, 54, 383-388. [CrossRef]

10. Zhu, Z.W.; Zhou, X.Q.; Liu, Z.W.; Wang, R.Q.; Zhu, L. Development of a piezoelectrically actuated two-degree-of-freedom fast tool servo with decoupled motions for micro-/nanomachining. Precis. Eng. 2014, 38, 809-820. [CrossRef]

11. Yang, Y.; Chen, S.; Huo, D.; Cheng, K. Performance analysis and optimal design of fast tool servo used for machining microstructured surfaces. Process. Inst. Mech. Eng. Part C 2008, 222, 1541-1546. [CrossRef]

12. Zhu, Z.W.; Zhou, X.Q.; Liu, Q.; Zhao, S.X. Multi-objective optimum design of fast tool servo based on improved differential evolution algorithm. J. Mech. Sci. Technol. 2011, 25, 3141-3149. [CrossRef]

13. Zhuang, C.; Xu, M.; Xiong, Z. Multi-objective topology optimization of compliant mechanism for fast tool servo. In Proceedings of the IEEE/ASME International Conference on Advanced Intelligent Mechatronics (AIM), Wollongong, Australia, 9-12 July 2013.

14. Rakuff, S.; Cuttino, J.F. Design and testing of a long-range, precision fast tool servo system for diamond turning. Precis. Eng. 2009, 33, 18-25. [CrossRef]

15. Lu, X. Electromagnetically-Driven Ultra-Fast Tool Servos for Diamond Turning. Ph.D. Thesis, Massachusetts Institute of Technology, Cambridge, MA, USA, 2005.

16. Wu, D.; Xie, X.D.; Zhou, S.Y. Design of a normal stress electromagnetic fast linear actuator. IEEE Trans. Magn. 2010, 46, 1007-1014. 
17. Fang, F.Z.; Nie, Y.H.; Zhang, X.D. Design of fast tool servo system based on magnetic field analysis. Nanotechnol. Precis. Eng. 2011, 9, 539-544.

18. Lee, W. Prediction of microcutting force variation in ultra-precision machining. Precis. Eng. 1990, 12, $25-28$. [CrossRef]

19. Smith, S.T. Flexures: Elements of Elastic Mechanisms; Gordon and Breach Science Publishers: Perth, Australia, 2000.

20. Nie, Y.H.; Fang, F.Z.; Zhang, X.D. System design of Maxwell force driving fast tool servos based on model analysis. Int. J. Adv. Manuf. Technol. 2014, 72, 25-32. [CrossRef]

21. Franklin, G.F.; David, J.P.; Abbas, E.-N. Feedback Control of Dynamics Systems; Pretince Hall Inc.: Upper Saddle River, NJ, USA, 2006.

22. Schittkowski, K.; Zillober, C.; Zotemantel, R. Numerical comparison of nonlinear programming algorithms for structural optimization. Struct. Multidiscip. Optim. 1994, 7, 1-19. [CrossRef]

23. Grace, A.C.W. Computer-Aided Control System Design Using Optimization Techniques. Ph.D. Thesis, University of Wales, Bangor, Gwynedd, UK, 1989.

24. Francisco, F.; Fischer, A.; Kanzow, C. On the accurate identification of active constraints. SIAM J. Optim. 1998, 9, 14-32.

25. Fletcher, R.; Leyffer, S.; Ralph, D.; Scholtes, S. Local convergence of SQP methods for mathematical programs with equilibrium constraints. SIAM J. Optim. 2006, 17, 259-286. [CrossRef]

26. Luo, Z. The Study of Sequential Quadratic Programming (SQP) Algorithms. Ph.D. Thesis, Guilin University of Electronic Technology, Guilin, China, 2008.

27. Hu, Q. Research on Sequential Quadratics Programming Method for Solving Constrained Optimization. Ph.D. Thesis, Hunan University, Hunan, China, 2008.

(C) 2017 by the authors. Licensee MDPI, Basel, Switzerland. This article is an open access article distributed under the terms and conditions of the Creative Commons Attribution (CC BY) license (http://creativecommons.org/licenses/by/4.0/). 